\title{
SCHOTTKY UNIFORMIZATIONS OF CLOSED RIEMANN SURFACES WITH ABELIAN GROUPS OF CONFORMAL AUTOMORPHISMS
}

\author{
by RUBÉN A. HIDALGO $\dagger$
}

(Received 8 June, 1992)

1. Introduction Let us consider a pair $(S, H)$ consisting of a closed Riemann surface $S$ and an Abelian group $H$ of conformal automorphisms of $S$. We are interested in finding uniformizations of $S$, via Schottky groups, which reflect the action of the group $H$. A Schottky uniformization of a closed Riemann surface $S$ is a triple $(\Omega, G, \pi: \Omega \rightarrow S)$ where $G$ is a Schottky group with $\Omega$ as its region of discontinuity and $\pi: \Omega \rightarrow S$ is a holomorphic covering with $G$ as covering group. We look for a Schottky uniformization $(\Omega, G, \pi: \Omega \rightarrow S)$ of $S$ such that for each transformation $h$ in $H$ there exists an automorphisms $t$ of $\Omega$ satisfying $h \circ \pi=\pi \circ t$.

In [7] we have obtained necessary conditions, called the condition $(A)$, to get a Schottky uniformization as desired. These necessary conditions involve only the action of $H$ at the set of fixed points of its non-trivial elements. In particular, if $H$ acts without fixed points, then it satisfies automatically such a condition. We show that in the case of $H$ Abelian, condition (A) is also sufficient.

An equivalent way to describe our problem in the language of three-manifolds is the following. Let $V$ be a handle-body of genus $g$ and let $S$ be the boundary of $V$. The surface $S$ is a closed orientable surface of genus $g$. Denote by $\operatorname{Diff}(S)$ the group of orientation preserving diffeomorphisms of $S$. Let $H$ be a finite abelian subgroup of $\operatorname{Diff}(S)$. We ask for the existence of an element $f$ of $\operatorname{Diff}(S)$ such that the group $f H f^{-1}$ extends to a group of orientation preserving diffeomorphisms of $V$. In the rest of our work we consider the Schottky group description of our problem.

In $1980 \mathrm{~L}$. Keen [11] discussed this problem for hyperelliptic Riemann surfaces $S$ with $H$ being the group generated by the hyperelliptic involution. In [5] and [6] we gave a similar discussion for closed Riemann surfaces which admit a general conformal involution. In [7] we discuss this problem for more general groups and we obtain necessary conditions, called the condition (A), to find a Schottky group as desired. In $[8,9,10]$ we have proved that condition (A) is sufficient if $H$ is isomorphic to a cyclic group or to the group $\mathbb{Z} / 2 \mathbb{Z} \oplus \mathbb{Z} / 2 \mathbb{Z}$ or to the Dihedral group $\mathbb{D}_{2 p}$ of order $2 p$ with $p$ a prime.

In general, if $S$ is a closed Riemann surface of genus $g \geq 2$ and $H$ is a group of conformal automorphisms of $S$ satisfying the condition (A), it is not true that this condition is sufficient.

2. Necessary conditions. In this section, we recall the definition of Schottky groups (Schottky uniformizations) and we get necessary conditions to be satisfied by the group $H$ to find a Schottky group as desired. At the end of the section we establish the main result of this work, which shows that these necessary conditions are also sufficient if the group $H$ is Abelian. For most of the definitions, concerning Kleinian groups, [14] is a good reference. 
We denote by $\hat{\mathbb{C}}=\mathbb{C} \cup\{\infty\} \cong \mathbb{C P}_{1}$ the Riemann Sphere. The group of conformal automorphisms of $\hat{\mathbb{C}}$ is the Möbius Group, also called the Fractional Linear Group, and denoted by $\mathbb{M}$.

Definition 1 (Schottky group of genus $g$ ). For $g \geq 1$, let $C_{k}, C_{k}^{\prime}, k=1, \ldots, g$, be $2 g$ Jordan curves on the Riemann sphere, $\mathbb{C}=\mathbb{C} \cup\{\infty\}$, such that they are mutually disjoint and bound a $2 g$-connected domain, say $D$. Suppose that for each $k$ there exists a fractional linear transformation $A_{k}$ with the following properties:

(i) $A_{k}\left(C_{k}\right)=C_{k}^{\prime}$;

(ii) $A_{k}$ maps the exterior of $C_{k}$ onto the interior of $C_{k}^{\prime}$.

The transformations $\left\{A_{i}: i=1, \ldots, g\right\}$ generate a subgroup $G$ of Möbius transformations, necessarily Kleinian, and $D$ is a fundamental domain for $G$, called a standard fundamental domain for $G$. This group is called a Schottky group of genus $g$.

Observe that if $G$ is a Schottky group, then $G$ is a free group on $g$ generators and all its elements, except the identity, are loxodromic [15]. These properties in fact define the Schottky groups of genus $g$, for $g \geq 1$. For our purposes, we define the Schottky group of genus zero to be the group with the identity as its only element, that is, the trivial group.

TheOrem 1 [15]. Let $G$ be a Kleinian group. Then $G$ is a Schottky group if and only if $G$ is purely loxodromic, finitely generated and free.

TheORem 2 [3]. If $G$ is a Schottky group, then corresponding to any set of free generators these exists a fundamental domain $D$, as above, whose boundary curves are identified by the given generators.

Definition 2. If $G$ is a Schottky group and $A_{1}, \ldots, A_{g}$ form a set of free generators, we say that $G=\left\langle A_{1}, \ldots, A_{g}\right\rangle$ is a marked Schottky group, and that the set of transformations $A_{1}, \ldots, A_{g}$ is a marking of $G$.

Let us remark that if $G$ is a Schottky group of genus $g$, then $\Omega(G) / G$ is a closed Riemann surface of genus $g$. Moreover, if $A_{1}, \ldots, A_{g}$ form a set of free generators for $G$ and $D$ is a standard fundamental domain for these generators with boundary curves $C_{k}$, $C_{k}^{\prime}, k=1, \ldots, g$, then these loops project to a set of $g$ disjoint homologically independent simple loops on $S$. Reciprocally, the retrosection theorem [2] say to us that we can reverse this situation.

THEOREM 3 (Retrosection theorem). Every closed Riemann surface $S$ of genus $g$ can be represented as $\Omega(G) / G, G$ being a Schottky group of genus $g$ with region of discontinuity $\Omega(G)$. More precisely, given a set of $g$ disjoint, homologically independent, simple closed curves $\gamma_{1}, \ldots, \gamma_{g}$ on $S$, one can choose $G$, and g generators $A_{1}, \ldots, A_{g}$ of $G$, so that there is a standard fundamental domain $D$ for $G$, bounded by curves $C_{1}$, $C_{1}^{\prime}, \ldots, C_{g}, C_{g}^{\prime}$ with $A_{i}\left(C_{i}\right)=C_{i}^{\prime}$, such that $\gamma_{i}$ is in the free homotopy class of the image of $C_{i}$ under $\Omega(G) \rightarrow \Omega(G) / G$. The marked Schottky group $G=\left\langle A_{1}, \ldots, A_{g}\right\rangle$ is determined by $\left(S, \gamma_{1}, \ldots, \gamma_{g}\right)$ except for replacing $A_{1}, \ldots, A_{g}$ by $B A_{1}^{n_{1}} B^{-1}, \ldots, B A_{g}^{n_{g}} B^{-1}$, where $B$ is a fractional linear transformation and $n_{i} \in\{-1,1\}$.

Remark 1. This theorem was first stated by Felix Klein in 1883 [12] and proved rigorously by Koebe [13] much later. Let us remark that an easy proof of this theorem can be given using Bers ideas on quasiconformal mappings [2]. 
DEFINITION 3. A Schottky uniformization of a closed Riemann surfaces of genus $g$ is a triple $(\Omega, G, \pi: \Omega \rightarrow S$ ) where, $G$ is a Schottky group (necessarily of genus $g$ ) with $\Omega$ as its region of discontinuity, and $\pi: \Omega \rightarrow S$ is a holomorphic covering, with $\operatorname{Deck}(\pi)=G$.

Definition 4. Let $(\Omega, G, \pi: \Omega \rightarrow S)$ be a Schottky uniformization of a Riemann surface $S$. Let $H$ be a group of conformal automorphisms of $S$. We say that $H$ lifts to this uniformization if each automorphism $h \in H$ lifts to a conformal automorphism of $\Omega$ under the covering $\pi: \Omega \rightarrow S$, that is, for each $h \in H$ there exists $t$ a conformal automorphism of $\Omega$ satisfying $\pi \circ t=h \circ \pi$. Observe that such a $t$ is necessarily a Möbius transformation [1].

Definition 5. For $p \in S$, the stabilizer of $p$ with respect to $H$ is the group

$$
H(p)=\{h \in H: h(p)=p\} .
$$

For the next definition, we need a classical result. Let $h \in H$ and $p \in S$ be as before such that $h(p)=p$. We can find a local coordinate system $(U, \phi)$ such that $\phi(p)=0$ and $\phi \circ h \circ \phi^{-1}(z)=e^{i \alpha} z$, for all $z \in \phi(U)$. Moreover, we can assume $\phi(U)=\Delta$, where $\Delta$ denotes the unit disc in the complex plane $\mathbb{C}$. The angle $\alpha=\alpha(h, p)$ is well defined up to a multiple of $2 \pi$, independent of the local coordinate and $\alpha\left(h^{k}, p\right)=k \alpha(h, p)$.

Definition 6 (The rotation number). Let $h \in H$ and $p \in S$ be such that $h(p)=p$. We normalize $\alpha$ by assuming that $-\pi<\alpha \leq \pi$. We will call $\alpha=\alpha(h, p)$ the rotation number of $h$ at $p$.

Assume the finite group $H$ lifts to some Schottky uniformization $(\Omega, G, \pi: \Omega \rightarrow S)$ of $S$. Let $K$ be the group obtained by the lifting of $H$. This group contains the Schottky group $G$ as a normal subgroup of finite index. In particular, the region of discontinuity of $K$ is also $\Omega$. It is easy to see that $K$ is a finitely generated, geometrically finite, function group without parabolic elements.

Maskit's classification of finitely generated function groups [17] asserts that $K$ is constructed by use of the Klein-Maskit Combination theorems from the following basic function groups:

(i) Finite groups.

(ii) Euclidean groups.

(iii) Finite extensions of cyclic loxodromic groups.

(iv) Quasi-Fuchsian groups.

(v) Degenerated groups.

The above properties of $K$ imply that we cannot use the groups of type (ii), (iv) or (v). So the group $K$ is constructed from groups of type (i) and (iii).

An easy consequence of the above is the following.

Proposition 1. Let $h$ be any elliptic element of $K$ with fixed points $p$ and $q$. Then (1) Either $p$ and $q$ are in $\Omega$ or there is an element of $G$ commuting with $h$. (2) If both fixed points of $h$ are in $\Omega$ and they are equivalent under $K$, then there is an involution $j$ in $K$ permuting them.

Proof. If $K$ is torsion free, then there is nothing to check. Let us assume $K$ has torsion. Let $h$ be any elliptic element of $K$ with $x$ and $y$ as its fixed points. If both points are regular points we are done. Let us assume $y$ is a limit point. Let $j$ be a primitive elliptic element in $K$ fixing $y$. 
CLAIM (i) $x$ is also a fixed point of $j$.

(ii) If $g(y)=y$, some $g$ in $K$, then either $g$ is conjugate in $K$ to a power of $j$ or $g$ is a loxodromic element with $x$ and $y$ as fixed points.

Proof. (Claim) (i) If $j(x) \neq x$, then the commutator $[j, h]=j h j^{-1} h^{-1}$ will be a parabolic element in $K$ with $y$ as fixed point. Since $K$ cannot have parabolic elements we must have $j(x)=x$ as claimed.

(ii) Let $g$ in $K$ be such that $g(y)=y$. The only possibility for $g$ is to be elliptic or loxodromic. By our assumption on $y$, we obtain that necessarily $g(x)=x$; otherwise $[g, j]$ will be a parabolic element of $K$ fixing the point $y$. At this point, $g$ is either a power of $j$, or a loxodromic element with $x$ and $y$ as fixed points. This ends the proof of our claim.

Now we continue with the proof of Proposition 1. Let $L$ be the geodesic in $\mathbb{H}^{3}$ with $x$ and $y$ as end points. The transformation $j$ acts as the identity on $L$.

Let $P$ be a convex fundamental polyhedron for $G$. Since $y$ is a limit point, which is not a parabolic fixed point, it must be a point of approximation for $G$ (see page 128 in [14]). This implies that $y$ cannot be in the closure of $P$ (see page 122 in [14]).

By the above observation, we can find a sequence of points $y_{n} \in L$, converging to $y$, all of them non-equivalent points under $K$, and a sequence $g_{n} \in K$, all of them different, such that $g_{n}\left(y_{n}\right)=z_{n} \in \bar{P}$, where $\bar{P}$ denotes the Euclidean closure of $P$.

Let us consider a subsequence such that $z_{n}$ converges, say to $z, g_{n}(y)$ converges, say to $u$, and $g_{n}(x)$ converges, say to $t$. In particular, the point $u$ and $t$ are limit points for the group $G$.

Since $z_{n} \in \bar{P}$, we have $z \in \bar{P}$. We have two possibilities for $z$, that is, $z$ is as regular point, or $z$ is a parabolic fixed point (see page 128 in [14]). Since $K$ does not have parabolic elements, $z$ is a regular point.

It is clear that the $z_{n}$ are elliptic fixed points, in fact $z_{n}=g_{n} j g_{n}^{-1}\left(z_{n}\right)$. This implies that $z_{n}$ must be in some edge of $P$. Since $P$ has only a finite number of edges, we can assume all $z_{n}$ lie on the same edge of $P$. Let $M$ be the geodesic in $\mathbb{t}^{3}$ containing this edge. In particular, $z$ must belong to the closure of $M$.

Let us consider the geodesics $L_{n}=g_{n}(L)$ through $z_{n}$, and having end points $g_{n}(x)$ and $g_{n}(y)$. Since we have supposed $g_{n}(x)$ and $g_{n}(y)$ to converge to $t$ and $u$, respectively, then $L_{n}$ converges either to a point or to the geodesic with end points $u$ and $t$. If $L_{n}$ converges to a point we necessarily have $u=t=z$, a contradiction to the fact that $z$ is regular point and $u$ is a limit point. The other possibility is that $L_{n}$ converges to a geodesic $\gamma$, with end points $u$ and $t$. In this case, since the end points of $\gamma$ are limit points and $z$ is known to be a regular point we must have $z$ in $\gamma \cap \mathbb{Q}^{3}$.

Any neighbourhood of $z$ contains $z_{n}$, for $n$ sufficiently large. Since $z$ is a regular point, there exists a neighborhood of $z$ which is precisely invariant by the elements of $G$ that fixes $z$, which is known to be finite. We can then assume without lost of generality that $g_{n} j g_{n}^{-1}(z)=z$, and $g_{n} j g_{n}^{-1}=h$. In other words, $\left(g_{m}^{-1} g_{n}\right) j\left(g_{m}^{-1} g_{n}\right)^{-1}=j$. Since $g_{n} j g_{n}^{-1}\left(z_{n}\right)=z_{n}, g_{n} j g_{n}^{-1}(z)=z$, and $z_{n} \neq z$ for all $n$, we have $g_{n} j g_{n}^{-1}(w)=w$, for all $w$ in $\gamma$. In particular, $g_{n} j g_{n}^{-1}(t)=t$ and $g_{n} t_{n}^{-1}(u)=u$. It follows that $\left\{g_{n}(x), g_{n}(y)\right\}=\{t, u\}$. The facts that $t \neq u$ and $g_{n}(x)$ converges to $t$ imply that $g_{n}(x)=t$ and $g_{n}(y)=u$, for $n$ sufficiently large. We may assume it holds for every $n$. The last observation implies that $g_{m}^{-1} g_{n}(x)=x$ and $g_{m}^{-1} g_{n}(y)=y$, for all $n, m$. 
The transformations $g_{m}^{-1} g_{n}$ also keep $L$ invariant, and for $n \neq m$ this transformation cannot be the identity on $L$. This implies that $g_{m}^{-1} g_{n}$ is a loxodromic element of $K$ with $x$ and $y$ as fixed points.

Proposition 1 implies some conditions on the set of points fixed by some non-trivial element of $H$ that we describe below.

THE CONDITION (A). The fixed points of the non-trivial elements of $H$ can be paired in the following way.

(A1) If $(p, q)$ is a pair, then $p \neq q, H(p)=H(q)$, and $\alpha(h, p)=-\alpha(h, q)$, for all $h \in H(p)$ of order greater than two.

(A2) If $(p, q)$ and $(r, s)$ are two different pairs, then $\{p, q\} \cap\{r, t\}=\phi$.

(A3) If $(p, q)$ is a pair and $h$ is in $H$, then $(h(p), h(q))$ is also a pair.

(A4) If $(p, q)$ is a pair with $p$ and $q$ equivalent under $H$, then there is an involution $J$ in $H$ permuting them.

Remark 2. (1) Observe that if we have a pairing satisfying conditions (A1), (A2) and (A4), then it is easy to get another pairing satisfying (A1)-(A4). (2) If the group $H$ is Abelian and $(p, q)$ is a pair as above with $p$ and $q$ equivalent under $H$, then $H(p)=H(q)$ is just the cyclic group in two elements.

The following shows an example of a closed Riemann surface of genus three, non-hyperelliptic, with an automorphism $h$ of order three with five fixed points. In particular, the cyclic group of order five generated by $h$ does not satisfy the condition (A). Let us consider the quartic complex curve in the complex projective plane $\mathbb{C} P^{2}$ given by the zero locus of

$$
a X^{4}+b Y^{4}+c X Y^{3}+d X^{2} Y^{2}+e X^{3} Y+f Z^{3} X+g Z^{3} Y=0 .
$$

for suitable complex numbers $a, b, c, d, e, f$ and $g$ (e.g. $a=b=f=1, c=d=e=g=0$ ), this quartic is non-singular and irreducible. Such a quartic is a non-hyperelliptic closed Riemann surface of genus three, admitting the automorphism of order three $h$ induced by the linear transformation

$$
h=\left(\begin{array}{ccc}
1 & 0 & 0 \\
0 & 1 & 0 \\
0 & 0 & \omega
\end{array}\right),
$$

where $\omega^{2}+\omega+1=0$. It is easy to check that this automorphism has in fact only five fixed points on the above Riemann surface. Now it is clear that the cyclic group generated by $h$ cannot sastisfy the condition (A).

The following is the main result of this paper.

Main THeorem. Let $S$ be a closed Riemann surface and let $H$ be an Abelian group of conformal automorphisms of $S$. Then condition $(A)$ is necessary and sufficient to find a Schottky uniformization $(\Omega, G, \pi: \Omega \rightarrow S)$ of $S$ for which $H$ lifts.

Let us recall a couple basic results of covering spaces. The proof of the two propositions below are quite simple and they are left to the reader as an exercise.

Proposition 2. Let $S$ be a closed Riemann surface of genus $g$ and let $H$ be a group of conformal automorphisms of $S$. If there exists a set of $g$ homologically independent disjoint 
simple loops on $S$, whose normalizer in $\Pi_{1}(S)$ is invariant under the action of $H$, then every element of $H$ lifts to a conformal automorphism of the region of discontinuity of the Schottky group (Schottky uniformization) defined, up to conjugation, by these g loops.

Proposition 3. Let $S$ be a closed Riemann surface of genus $g$ and let $\left\{\alpha_{k}: k=\right.$ $1, \ldots, t\}$ be a family of disjoint simple loops on $S$. Let us assume that $S-\bigcup_{k=1}^{t} \alpha_{k}$ is a disjoint union of surfaces of genus zero with boundaries. Then there exist $g$ loops in this family, say $\alpha_{1}, \ldots, \alpha_{g}$, such that $S-\bigcup_{k=1}^{g} \alpha_{k}$ is a sphere with $2 g$ deleted discs. In particular, they are homologically independent and the normalizer in $\Pi_{1}(S)$ of these $g$ loops is the same as for all the family.

To show our main theorem, we construct a set of loops satisfying the hypotheses of the above two propositions. It is done in the next section.

3. Proof of the Main Theorem. Let $S$ be a closed Riemann surface and let $H$ be a finite Abelian group of conformal automorphisms of $S$ satisfying the condition (A). Assume $H$ has order $N$ and let us pair the fixed points of the non-trivial elements of $H$ satisfying (A1)-(A4) of condition (A).

Denote by $S / H$ the quotient Riemann surface and by $\pi: S \rightarrow S / H$ the natural holomorphic branched covering induced by the action of $H$ on $S$. The genus of $S$ and $S / H$ will be denoted by $g$ and $\gamma$, respectively. The subset of $S / H$ consisting of the branch values of $\pi$ will be denoted by $\mathscr{B}$. The branch values of $\pi$ are exactly the projection to $S / H$ of the fixed points of the non-trivial elements of $H$. In case that $\mathscr{B}$ is not empty, we can write

$$
\mathscr{B}=\left\{P_{1}, Q_{1}, P_{2}, Q_{2}, \ldots, P_{t}, Q_{t}, Z_{1}, \ldots, Z_{m}\right\},
$$

where for each $j \in\{1, \ldots, t\}$, there exists a pair as defined above, say $\left(p_{j}, q_{j}\right)$, such that $\pi\left(p_{j}\right)=P_{j}$ and $\pi\left(q_{j}\right)=Q_{j}$, and for each $k \in\{1, \ldots, m\}$, there exists a pair $\left(r_{k}, s_{k}\right)$ such that $\pi\left(r_{k}\right)=\pi\left(s_{k}\right)=Z_{k}$.

Condition (A) and the fact that $H$ is abelian imply that the stabilizer of each point over $Z_{k}$ is the same group in two elements $\mathbb{Z} / 2 \mathbb{Z}$. In particular, for each pair $(r, s)$ such that $\pi(r)=\pi(s)$ we have that the involution fixing $r$ and $s$ and the involution that permutes them generate the Klein group $\mathbb{Z} / 2 \mathbb{Z} \oplus \mathbb{Z} / 2 \mathbb{Z}$.

Clearly, if the liftings of $Z_{k}$ and $Z_{l}$ have the same involution as stabilizer, then we can change our pairing still satisfying the condition (A) in the following way. Let $(r, s)$ and $(t, u)$ be two pairs such such that $\pi(r)=\pi(s)=Z_{k}$ and $\pi(t)=\pi(u)=Z_{l}$. Pair $r$ with $t$ and $s$ with $u$. Now use the action of $H$ to pair $h(r)$ with $h(t)$ and to pair $h(s)$ with $h(u)$, for all $h$ in $H$. Now we can assume that the liftings of different $Z_{k}$ have different involutions as stabilizer. From now on, we assume this is the case.

For each $j \in\{1, \ldots, t\}$ and each $k \in\{1, \ldots, m\}$, let us write

(1) $\pi^{-1}\left(P_{j}\right)=\left\{p_{j}^{i} ; i_{j}=1, \ldots, l_{j}\right\}$;

(2) $\pi^{-1}\left(Q_{j}\right)=\left\{q_{j}^{i_{j}} ; i_{j}=1, \ldots, l_{j}\right\}$; and

(3) $\pi^{-1}\left(Z_{k}\right)=\left\{r_{k}^{v_{k}}, s_{k}^{v_{k}}, v_{k}=1, \ldots, N / 4\right\}$,

where $l_{k}$ divides $N$, and $\left(p_{j}^{i_{j}}, q_{j}^{i_{j}}\right)$ is a pair. Denote by $n_{j}=N / l_{j}$. 
For each $j \in\{1, \ldots, t\}$ and each $i \in\left\{1, \ldots, l_{j}\right\}$, let $F_{j}^{i} \in H$ be such that $0<$ $\alpha\left(F_{j}^{i}, p_{j}^{i}\right) \leq\left\|\alpha\left(h, p_{j}^{i}\right)\right\|$, over all $h \in H\left(p_{j}^{i}\right)-\{I\}$. Clearly, $\alpha\left(F_{j}^{i}, p_{j}^{i}\right)=2 \pi / n_{j}$.

For each $k \in\{1, \ldots, m\}$, denote by $h_{k}$ the involution which stabilizes one and therefore every point in $\pi^{-1}\left(Z_{k}\right)$.

The Riemann-Hurwitz formula [4] gives us the following relation

$$
g=N(\gamma+t+m / 4-1)+1-\sum_{j=1}^{t} l_{j}
$$

We proceed to construct a set of disjoint simple loops on $S$ such that they are invariant as set of loops under the action of $H$ and they decompose the surface $S$ into spheres with holes.

Let us construct a system of oriented simple loops as follows. If $\gamma \geq 1$, let $\alpha_{i}$ and $\beta_{i}$, $i=1, \ldots, \gamma$, be a set of oriented simple loops on $S / H-\mathscr{B}$ satisfying the following:

(1) $\alpha_{i} \cap \mathscr{B}=\phi$, for all $i=1, \ldots, \gamma$;

(2) $\beta_{i} \cap \mathscr{B}=\phi$, for all $i=1, \ldots, \gamma$;

(3) $\alpha_{i} \cap \alpha_{j}=\phi$, for all $i \neq j$;

(4) $\alpha_{i} \cap \beta_{j}=\phi$, for all $i \neq j$;

(5) $\beta_{i} \cap \beta_{j}=\phi$, for all $i \neq j$;

(6) $\alpha_{i} \cap \beta_{i}$ consists of exactly one point, for all $i=1, \ldots, \gamma$; and

(7) $\alpha_{i}, \beta_{i}=+1$, for all $i=1, \ldots, \gamma$, where . denotes the intersection number (see Figure 1).

Let us consider a set of simple loops (oriented in the natural way) $\eta_{i}, i=1, \ldots, \gamma$, such that

(8) $\eta_{i}$ is homotopic to the commutator $\alpha_{i} \beta_{i} \alpha_{i}^{-1} \beta_{i}^{-1}$ on $S / H-\mathscr{B}$; and

(9) $\eta_{i} \cap \mathscr{B}=\phi$ (see Figure 1).

Let us denote by $T_{k}$ the torus with boundary $\eta_{i}$ obtained by cutting $S / H$ along the $\eta_{k}$ loops, for $k=1, \ldots, \gamma$ (see Figure 1 ).

If $t \geq 1$, we consider a set of disjoint simple loops, $\delta_{j}, j=1, \ldots, t$, on $S / H-\mathscr{B}$ satisfying the following properties.

(10) If $\gamma \geq 1$, then $\delta_{j}$ is disjoint from the loops $\alpha_{i}(i=1, \ldots, \gamma)$ and $\eta_{k}(k=1, \ldots, \gamma)$ constructed above, for all $j=1, \ldots, t$;

(11) $\delta_{j}$ bounds a conformal disc containing the points $P_{j}$ and $Q_{j}$ and no other branch value of $\pi$, for all $j=1, \ldots, t$.

Let us denote by $\Delta_{j}$ the conformal disc bounded by the loop $\delta_{j}$, for $j=1, \ldots, t$ (see Figure 1).

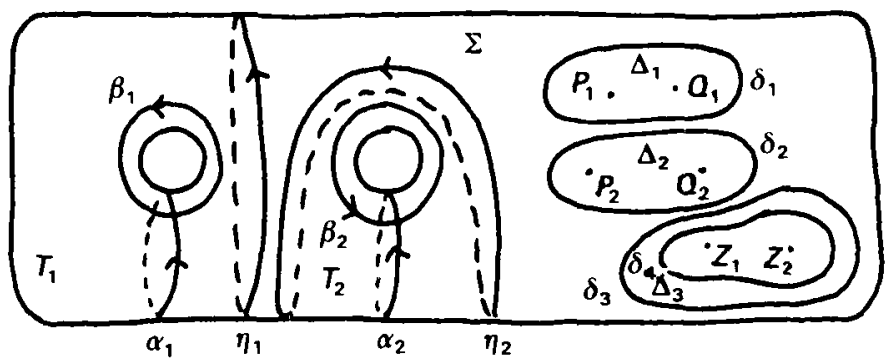

Figure 1: $\gamma=2, t=2$ and $m=3$. 
If $m \geq 1$, let $\delta_{t+1}$ be a simple loop disjoint from the above ones and bounding a disc $\Delta_{t+1}$ containing in its interior the branch values $Z_{k}$, for all $k$ (see Figure 1).

If $m$ is odd, we construct a simple loop $\delta_{t+2}$ inside the disc $\Delta_{t+1}$, such that this loop bound a disc containing in its interior the branch values $Z_{k}, k \in\{1, \ldots, m-1\}$. Denote by $\sigma$ surface bounded by the loops $\delta_{t+1}$ and $\delta_{t+2}$ (see Figure 1).

In the disc bounded by the loop $\delta_{t+2}$ if $m$ is odd, or by the loop $\delta_{t+1}$ otherwise, we construct a set of disjoint simple loops $\phi_{r}, r=1, \ldots, T$, satisfying the following properties;

(I) If $m$ is even, say $m=2 M$;

there are $M$ simple loops, say $\phi_{k}$ for $k=1, \ldots, M$, each one bounding a disc $\Phi_{k}$ containing exactly two branch values.

(II) If $m$, is odd, say $m=2 M+1$;

there are $M$ simple loops, say $\phi_{k}$ for $k=1, \ldots, M$, each one bounding a disc $\Phi_{k}$ containing exactly two branch values.

(III) The rest of the loops are contained in the common region bounded by the above $M$ loops and the loop $\delta_{t+1}$ or $\delta_{t+2}$, and these loops dissect the above region into pants,

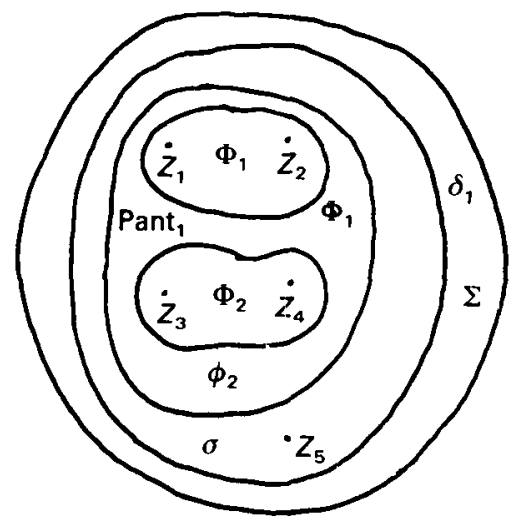

Figure 2: $\gamma=0, t=0$ and $m=5$.

denoted by Pant ${ }_{s}$, for $s=1, \ldots, K$ (see Figure 2 ).

We rename the branch values $z_{k}$ in such a way that the disc $\Phi_{k}$, bounded by $\phi_{k}$, contains $Z_{2 k-1}$ and $Z_{2 k}$. Clearly the group generated by all the involutions $h_{k}$ is isomorphic to a group of the form $\mathbb{Z} / 2 \mathbb{Z} \oplus \ldots \oplus \mathbb{Z} / 2 \mathbb{Z}=\bigoplus_{i=1}^{l} \mathbb{Z} / 2 \mathbb{Z}$, for some $l$.

Now, the loops $\alpha_{i}, \eta_{i}, \delta_{j}$ and $\phi_{r}$ dissect the surface $S / H$ into spheres with holes. The next step is to show that the liftings under $\pi$ of these loops define a set of disjoint simple loops on $S$ satisfying the desired conditions.

Let us call by $\Sigma$ the (connected) surface with boundary the loops $\eta_{i}$ and $\delta_{j}$, for $i=1, \ldots, \gamma$ and $j=1, \ldots, t+1$. This surface is sphere with $t+1+\gamma$ deleted discs.

Lemma 1. Each loop $\eta_{i}$ and $\delta_{j}$ lifts to a loop on $S$, for $i=1, \ldots, \gamma$ and for $j=1, \ldots, t+1$.

Proof. For fixed $i \in\{1, \ldots, \gamma\}$, the loop $\eta_{i}$ lifts to a loop if and only if the loop 
$\alpha_{i} \beta_{i} \alpha_{i}^{-1} \beta_{i}^{-1}$ lifts to a loop. Denote by $x$ the intersection of the loops $\alpha_{i}$ and $\beta_{i}$. If $z$ is any lifting of $x$, let us lift the loops $\alpha_{i}$ and $\beta_{i}$ at $z$. The end point of the lifting of $\alpha_{i}$ is $f(z)$ and the end point of the lifting of $\beta_{i}$ is $h(z)$, for some $f$ and $h$ in $H$. The end point of the lifting of $\alpha_{i} \beta_{i} \alpha_{i}^{-1} \beta_{i}^{-1}$ at $z$ is $h^{-1} f^{-1} h f(z)$. Since the group $H$ is abelian, this end point is again $z$, and we are done in this case. For fixed $j \in\{1, \ldots, t\}$, let $y$ be any point in $\delta_{j}$. Let us consider two disjoint (except at $y$ ) simple loops based at $y$, say $d_{1}$ and $d_{2}$, such that $d_{1}$ bounds a disc containing the point $P_{j}$ and $d_{2}$ bounds a disc containing the point $Q_{j}$. Orient these loops in such a way that going in the positive orientation the branch point they bound is at the left side. If we show that the loop $d_{1} d_{2}$ lifts to a loop we will be done. Let $\omega$ be a lifting of $y$ and let $f(\omega)$ and $h(\omega)$ the end points of the liftings of $d_{1}$ and $d_{2}$ at $\omega$ respectively. Since $P_{j}$ and $Q_{j}$ are projections of a pair, then necessarily $f=h^{-1}$. Now the end point of the lifting of $d_{1} d_{2}$ at $\omega$ is $f h(\omega)=f f^{-1}(\omega)=\omega$. Since the loop $\delta_{t+1}$ is homotopic to the product of the $\eta_{i}$ and $\delta_{j}$ loops, for $i=1, \ldots, \gamma$ and $j=1, \ldots, t$, then it must lift to a loop on $S$.

If $m$ is odd, then clearly the loop $\delta_{t+2}$ cannot lift to a loop on $S$. But $\delta_{t+2}^{2}$ lifts to a loop on $S$. This is a consequence of the fact that $\delta_{t+2}$ is homotopic to the product of two small simple loops around $Z_{m}$ and $\delta_{t+1}$, respectively. Each loop $\phi_{r}$ either lifts to a loop or its square lifts to a loop. The last is a consequence of the fact that each loop $\phi_{r}$ is homotopic to the product of small simple loops around the points $Z_{k}$ contained in the disc bounded by it.

Definition 7. A circle domain is a region on a closed Riemann surface obtained as a component of cutting the closed surface along a finite number of pairwise disjoint rounded simple loops (in the natural hyperbolic, spherical or euclidean structure of the surface). We will use the following theorem due to B. Maskit.

Theorem 5 [16]. Let $S$ be a topologically finite Riemann surface of genus g. Then there exists a closed Riemann surface $\bar{S}$ of genus $g$, and there is a conformal embedding $f: S \rightarrow \bar{S}$ so that $f(S)$ is a circle domain on $\bar{S}$. This representation is unique; that is, if there is another closed Riemann surface $\bar{S}^{\prime}$, also of genus $g$, and there is a conformal embedding $f^{\prime}: S \rightarrow \bar{S}^{\prime}$, also of genus $g$, so that $f^{\prime}(S)$ is also a circle domain, then there is a conformal homeomorphism $h: \bar{S} \rightarrow \bar{S}^{\prime}$ with $f^{\prime}=h f$. Moreover, if $H$ is the group of conformal automorphisms of $S$, then $\mathrm{fH}^{-1}$ can be extended to a group of conformal automorphisms of $\bar{S}$.

Now we proceed to describe each component of the lifting via $\pi$ of each surface $T_{i}$, $\Delta_{j}, \Sigma, \sigma, \Phi_{k}$ and Pant $_{s}$.

LeMMA 2. $\pi^{-1}(\Sigma)=\coprod_{l=1}^{N} \Sigma_{l}$, where each $\Sigma_{l}$ is homeomorphic under $\pi$ to $\Sigma$.

Proof. Let $\Sigma_{l}$ be a component of $\pi^{-1}(\Sigma)$ and let us consider the restriction of $\pi$ to these surfaces

$$
\pi: \Sigma_{l} \rightarrow \Sigma \text {. }
$$

This is a holomorphic unbranched regular covering, with covering group $H_{l}$. Let us denote by $L_{l}$ the order of this group, so the above covering has degree $L_{l}$. Since each loop 
$\eta_{i}$ and $\delta_{j}$ lifts to a loop on $S$, we have that $\Sigma_{l}$ is a surface of genus $g_{l}$ with $L_{l}(t+\gamma+1)$ deleted discs. We can holomorphically embedd the surface $\Sigma$ in the Riemann sphere, $\hat{\mathbb{C}}$, as the complement of $(t+\gamma+1)$ deleted holomorphic discs, and we can holomorphically embedd the surface $\Sigma_{l}$ in a closed Riemann surface, $\tilde{\Sigma}_{l}$, of genus $g_{l}$ as the complement of $L_{l}(t+\gamma+1)$ deleted holomorphic discs. Now, we can extend the covering map $\pi$ to a regular unbranched covering

$$
\pi: \bar{\Sigma}_{l} \rightarrow \hat{\mathbb{C}}
$$

Let us apply the Riemann-Hurwitz formula to this covering. In this case, we obtain the following equality:

$$
g_{l}=1-L_{l}
$$

from where we obtain that $L_{l}=1$ and $g_{l}=0$.

Lemma 3. For each $j=1, \ldots, t, \pi^{-1}\left(\Delta_{j}\right)=\bigsqcup_{k=1}^{l i} \Delta_{j, k}$, where each $\Delta_{j, k}$ is a surface of genus zero with $n_{j}$ deleted discs, precisely invariant under the cyclic group generated by $F_{j}^{k}$ in $H$.

Proof. Let $\Delta_{j, k}$ be a component of $\pi^{-1}\left(\Delta_{j}\right)$ and let us consider the restriction of $\pi$ to these surfaces

$$
\pi: \Delta_{j, k} \rightarrow \Delta_{j}
$$

This is a holomorphic regular covering branched at $P_{j}$ and $Q_{j}$, with covering group $H_{j}$ and with the property that the loop $\delta_{j}$ lifts to a loop. Let us denote by $L_{j}$ the order of the group $H_{j}$, so the above covering has degree $L_{j}$. We have that $\Delta_{j, k}$ is a surface of genus $g_{j}$ with $L_{j}$ deleted discs. We can holomorphically embedd the surface $\Delta_{j}$ in the Riemann sphere, $\hat{\mathbb{C}}$, as the complement of a holomorphic disc, and we can holomorphically embedd the surface $\Delta_{j, k}$ in a closed Riemann surface, $\Delta_{j, k}$, of genus $g_{j}$ as the complement of $L_{j}$ holomorphic discs. Now, we can extend the covering map $\pi$ to a regular branched covering

$$
\pi: \tilde{\delta_{, k}} \rightarrow \hat{\mathbb{C}}
$$

with the same branching as before, that is, we do not add extra branch points to this covering. Let us apply the Riemann-Hurwitz formula to this covering. In this case, we obtain the following equality:

$$
g_{l}=1-L_{j} / n_{j}
$$

from where we obtain that $L_{j}=n_{j}$ and $g_{j}=0$. Now it is also clear that $H_{j}$ must be the cyclic group generated by $F_{j}^{k}$.

Lemma 4. $\pi^{-1}\left(T_{i}\right)=\coprod_{r=1}^{R_{i}} T_{i, r}$, where $R_{i}$ divides $N$ and $T_{i, r}$ is a torus with $N / R_{i}$ deleted discs. Moreover, $T_{i, r}-\left(\pi^{-1}\left(\alpha_{i}\right) \cap T_{i, r}\right)$ is the disjoint union of $N_{i}$ spheres with $\left(N / N_{i} R_{i}+2\right)$ deleted discs, where $N_{i} R_{i}$ divides $N$.

Proof. Let $T_{i, r}$ be a component of $\pi^{-1}\left(T_{i}\right)$ and let us consider the restriction of $\pi$ to these surfaces

$$
\pi: T_{i, r} \rightarrow T_{i}
$$


This is a holomorphic unbranched regular covering with covering group $H_{i}$ and with the property that the loop $\eta_{i}$ lifts to a loop. Let us denote by $L_{i}$ the order of the group $H_{i}$, so the above covering has degree $L_{i}$. We have that $T_{i, r}$ is a surface of genus $g_{i}$ with $L_{i}$ deleted discs. We can holomorphically embedd the surface $T_{i}$ in a torus, $\mathscr{T}_{\rho}$, as the complement of a holomorphic disc, and we can holomorphically embedd the surface $T_{i, r}$ in a closed Riemann surface, $\tilde{T}_{i, r}$, of genus $g_{i}$ as the complement of $L_{i}$ holomorphic discs. Now, we can extend the covering map $\pi$ to a regular unbranched covering

$$
\pi: \tilde{T}_{i, r} \rightarrow \mathscr{T}_{\rho} .
$$

Let us apply the Riemann-Hurwitz formula to this covering. In this case, we obtain the following equality:

$$
g_{i}=1,
$$

that is, $\tilde{T}_{i, r}$ is a torus. The group $H_{i}$ is a finite subgroup of conformal automorphisms of $T_{i, r}$ acting without fixed points. This group is known to be of the form $\mathbb{Z} / N_{i} \mathbb{Z} \oplus \mathbb{Z} /\left(N / N_{i} R_{i}\right) \mathbb{Z}$, for some $N_{i}$ and $R_{i}$, such that $N_{i} R_{i}$ divides $N$. The lemma follows as consequence of the above.

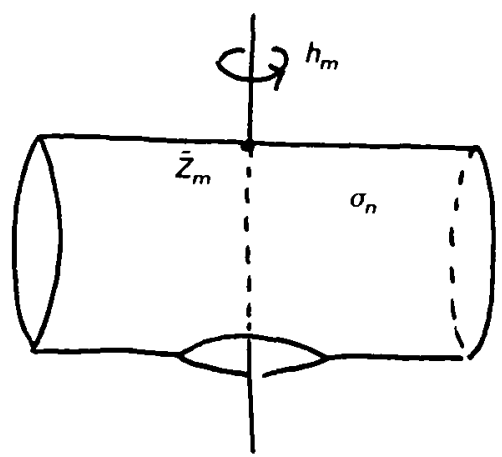

Figure 3.

LeMma 5. $\pi^{-1}(\sigma)=\coprod_{r=1}^{N / 2} \sigma_{r}$, where $\sigma_{r}$ is a sphere with 3 deleted discs and stabilizer in $H$ the group in two elements generated by the involution $h_{m}$ that fixes any lifting of $Z_{m}$. (See Figure 3.)

Proof. Let $\sigma_{r}$ be any component of $\pi^{-1}(\sigma)$. Let us restrict our covering $\pi$ to these parts, that is

$$
\pi: \sigma_{r} \rightarrow \sigma .
$$

This is a regular covering of degree $d$ with branch value $Z_{m}$ of order two. The loop $\delta_{t+1}$ lifts to a loop and the loop $\delta_{t+2}$ lifts to a path, but its square lifts to a loop on $\sigma_{r}$. The surface $\sigma_{r}$ is a surface of genus $g_{r}$ and with $(d+d / 2)$ holes. By Maskit's result, we may assume the surface $\sigma$ to be the Riemann sphere minus a holomorphic disc and the surface 
$\sigma_{r}$ to be the complement of $(d+d / 2)$ disjoint holomorphic discs on a closed Riemann surface $\tilde{\sigma}_{r}$ of genus $g_{r}$. We can extend our regular branch covering to a branch covering

$$
\pi: \tilde{\sigma}_{r} \rightarrow \hat{\mathbb{C}},
$$

with the same covering group and with two branch values of order two, one of them $Z_{m}$ and the other in the complement disc of $\sigma$ on the Riemann sphere $\hat{\mathbb{C}}$. Now, let us apply the Riemann-Hurwitz formula to this covering to obtain the equality

$$
g_{r}=1-d / 2 \text {. }
$$

As consequence, $g_{r}=0$ and $d=2$. Since the involution $h_{m}$ must be the covering group of this covering, we must have that this group is generated by the involution $h_{m}$.

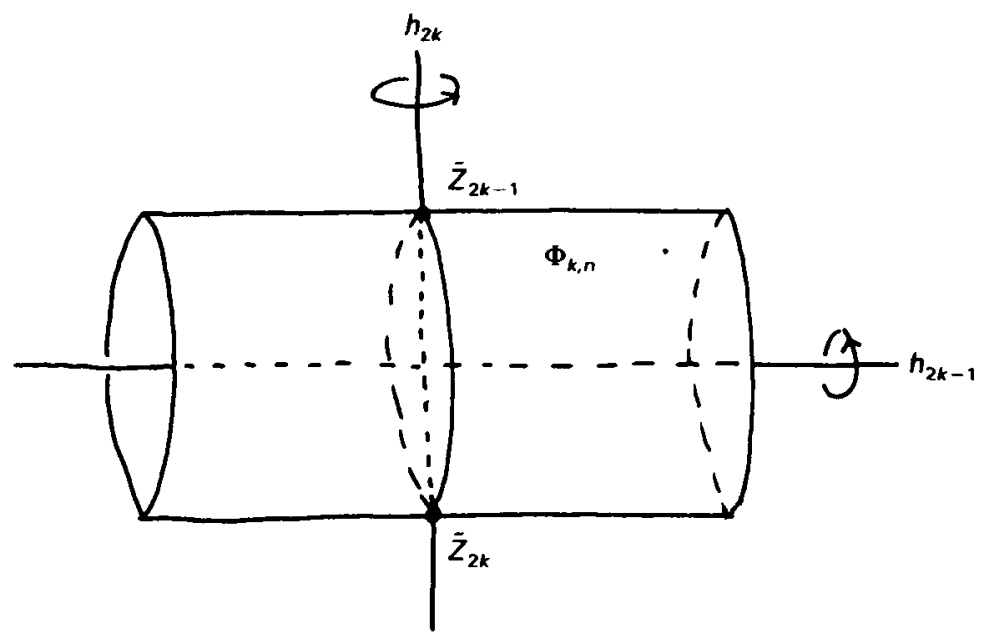

Figure 4.

LemMA 6. For each $k=1, \ldots, M, \pi^{-1}\left(\Phi_{k}\right)=\coprod_{r=1}^{N / 4} \Phi_{k, r}$, where $\Phi_{k, r}$ is a sphere with 2 holes an invariant under the Klein group generated by $h_{2 k-1}$ and $h_{2 k}$. (See Figure 4.)

Proof. Let $\Phi_{k, r}$ be any component of $\pi^{-1}\left(\Phi_{k}\right)$. Let us restrict our covering $\pi$ to these parts, that is

$$
\pi: \Phi_{k, r} \rightarrow \Phi_{k}
$$

This is a regular covering of degree $d$ with branch values $Z_{2 k-1}$ and $Z_{2 k}$ of order two. The loop $\phi_{k}$ lifts to a path, but its square lifts to a loop on $\Phi_{k, r}$. The surface $\Phi_{k, r}$ is a surface of genus $g_{k}$ and with $d / 2$ holes. By Maskit's result, we may assume the surface $\Phi_{k}$ to be the Riemann sphere minus a holomorphic disc and the surface $\Phi_{k, r}$ to be the complement of $d / 2$ disjoint holomorphic discs on a closed Riemann surface $\Phi_{k, r}^{\sim}$ of genus $g_{k}$. We can extend our regular branch covering to a branch covering

$$
\pi: \Phi_{k, r}^{\sim} \rightarrow \hat{\mathbb{C}},
$$


with the same covering group and with three branch values of order two, two of them $Z_{2 k-1}, Z_{2 k}$ and the other in the complementary disc of $\Phi_{k}$ on the Riemann sphere $\hat{\mathbb{C}}$. Now, let us apply the Riemann-Hurwitz formula to this covering to obtain the equality

$$
g_{k}=1-d / 4 \text {. }
$$

As a consequence, $g_{k}=0$ and $d=4$. Since the involutions $h_{2 k-1}$ and $h_{2 k}$ belong to the covering group and they generate a Klein group, then we must have that this group is exactly all the covering group.

For each $r$, denote by $P$ the pant Pant $r$ and by $\gamma_{1}, \gamma_{2}$ and $\gamma_{3}$ the boundary loops of $P=$ Pant $_{r}$. Since each of the loops $\gamma_{i}$ either lifts to a loop or its square lifts to a loop and the loop $\gamma_{3}$ is homotopic to the product of $\gamma_{1}$ and $\gamma_{2}$, we have three possibilities for the liftings of $P$.
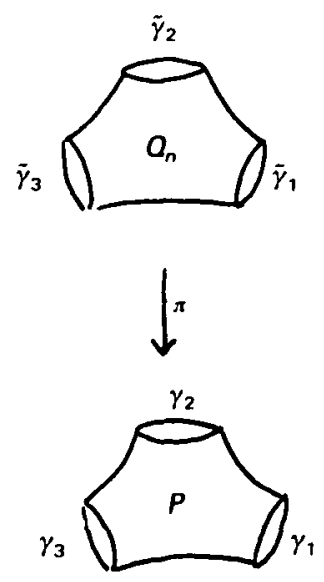

(1)

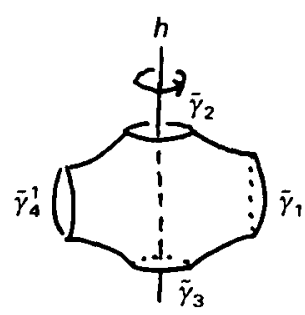

$\downarrow$

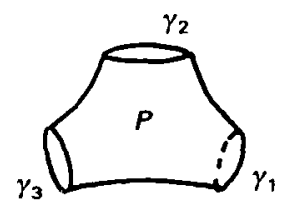

(2)
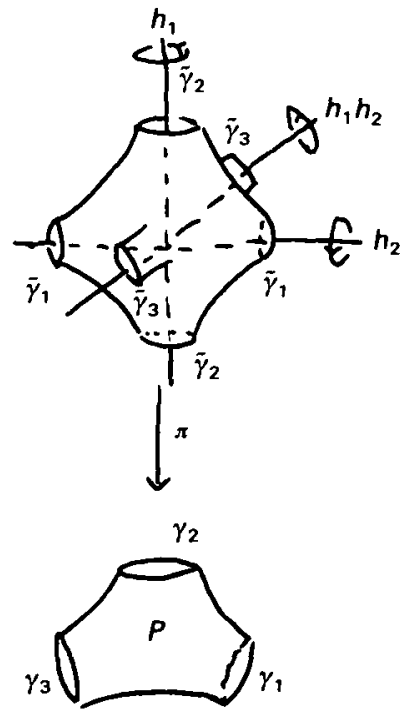

(3)

Figure 5 . Lemma 7. (1) If $\gamma_{1}$ and $\gamma_{2}$ lift to loops, then $\pi^{-1}(P)=\coprod_{r=1}^{N} Q_{r}$, where $Q_{r}$ is
biholomorphic to $P$ under $\pi$. In particular, $Q_{r}$ is a pant.

(2) If $\gamma_{1}$ lifts to a loop and $\gamma_{2}$ lifts to a path, then $\pi^{-1}(P)=\coprod_{r=1}^{N / 2} Q_{r}$, where $Q_{r}$ is a sphere with four holes. The subgroup of $H$ keeping $Q_{r}$ invariant is a group of order 2.

(3) If $\gamma_{1}$ and $\gamma_{2}$ both lift to paths, then either we are in case (2) by permuting the loops $\gamma_{1}$ and $\gamma_{3}$ or $\pi^{-1}(P)=\bigsqcup_{r=1}^{N / 4} Q_{r}$, where $Q_{r}$ is a sphere with six holes. In the last case, the subgroup of $H$ keeping invariant $Q_{r}$ is a Klein group. (See Figure 5.) 
Proof. (1) Let us assume the loops $\gamma_{i}$ lift to loops for $i=1$, 2. Since the loop $\gamma_{3}$ is homotopic to the product of them, then it lifts also to a loop. Let $Q_{r}$ be a component of $\pi^{-1}(P)$, and consider the regular unbranched covering

$$
\pi: Q_{r} \rightarrow P .
$$

This is a regular unbranched covering of degree $d$. The surface $Q_{r}$ is a surface of genus $g$ with $3 d$ deleted discs. Again, using Maskit's result we may assume that $P$ is the complement of three disjoint holomorphic discs in the Riemann sphere and $Q_{r}$ is the complement of $3 d$ disjoint holomorphic discs in a closed Riemann surface $\tilde{Q}_{r}$ of genus $g$. We can extend our covering to a regular unbranched one of degree $d$

$$
\pi: \tilde{Q}_{r} \rightarrow \hat{\mathbb{C}} .
$$

We apply the Riemann-Hurwitz formula to this covering to obtain the equality

$$
g=1-d .
$$

From this equality we obtain that $g=0$ and $d=1$.

(2) Let us assume the loop $\gamma_{1}$ lifts to loop and the loop $\alpha_{2}$ lifts to a path. Since the loop $\gamma_{3}$ is homotopic to the product of them, then it lifts also to a path. Let $Q_{r}$ be a component of $\pi^{-1}(P)$, and consider the regular unbranched covering

$$
\pi: Q_{r} \rightarrow P .
$$

This is a regular unbranched covering of degree $d$. The surface $Q_{r}$ is a surface of genus $g$ with $(d+d / 2+d / 2)=2 d$ deleted discs. Using Maskit's result we may assume that $P$ is the complement of three disjoint holomorphic discs in the Riemann sphere and $Q_{r}$ is the complement of $2 d$ disjoint holomorphic discs in a closed Riemann surface $\tilde{Q}_{r}$ of genus $g$. We can extend our covering to a regular one of degree $d$

$$
\pi: \tilde{Q}_{r} \rightarrow \hat{\mathbb{C}},
$$

With two branch values of order two. We apply the Riemann-Hurwitz formula to this covering to obtain the equality

$$
g=1-d / 2 .
$$

From this equality we obtain that $g=0$ and $d=2$.

(3) Let us assume the loops $\gamma_{i}$ lift to paths for $i=1,2$. Since the loop $\gamma_{3}$ is homotopic to the product of them, then it lifts either to a loop or to a path. In the first case, we permute the loops $\gamma_{1}$ and $\gamma_{3}$. In this way, we are in the previous case. Let us assume now that the three loops lift to paths. Let $f_{i}$ be the elements of $H$ which are determined in the following way. Take a point $x_{i}$ in $\gamma_{i}$ and let $u_{i}$ be a lifting of that point in $Q_{r}$. Lift the loop $\gamma_{i}$ at $u_{i}$ and look at its end point. That point has the form $f_{i}\left(u_{i}\right)$ for a unique $f_{i}$ in $H$. Since the group $H$ is abelian, the transformation $f_{i}$ is well defined. Also, since the loops lift to paths with the property that their square lift to loops, these elements are non-trivial and of order two. Since $\gamma_{3}$ is homotopic to the product of $\gamma_{1}$ and $\gamma_{2}$, then $f_{3}=f_{1} f_{2}$. In particular, the group generated by these elements is the Klein group. Let $Q_{r}$ be a component of $\pi^{-1}(P)$, then we consider the regular unbranched covering

$$
\pi: Q_{r} \rightarrow P \text {. }
$$


This is a regular unbranched covering of degree $d$. The surface $Q_{r}$ is a surface of genus $g$ with $3 d / 2$ deleted discs. Again, using Maskit's result we may assume that $P$ is the complement of three disjoint holomorphic discs in the Riemann sphere and $Q_{r}$ is the complement of $3 d / 2$ disjoint holomorphic discs in a closed Riemann surface $\tilde{Q}_{r}$ of genus $g$. We can extend our covering to a regular one of degree $d$ with three branch values of order two

$$
\pi: \tilde{Q}_{r} \rightarrow \hat{\mathbb{C}} .
$$

We apply the Riemann-Hurwitz formula to this covering to obtain the equality

$$
g=1-d / 4 \text {. }
$$

From this equality we obtain that $g=0$ and $d=4$. Since the Klein group has order 4 , then the covering group is exactly the one generated by the involutions $f_{i}$, for $i=1,2$.

As consequence of the above lemmas, we obtain that the liftings of the loops $\alpha_{i}, \eta_{i}$, $\delta_{j}$ and the loops $\phi_{r}$ are a family of disjoint simple loops on $S$ which dissect $S$ into spheres with holes. Now, we can proceed to finish the proof of the Main Theorem as consequence of Propositions 2 and 3 in Section 2. Denote by $\mathcal{M}$ the normalizer in $\Pi_{1}(S)$ of the liftings of the loops $\eta_{i}, \alpha_{i}^{N / N_{i} R_{i}}, \delta_{j}$ and $\phi_{r}$, for $i=1, \ldots, \gamma, j=1, \ldots, t$ and $r=1, \ldots, T$. Since $S$ minus the liftings of the above loops is a disjoint union of spheres with deleted discs, then there exists a sub-family $\mathcal{N}$ of the above liftings consisting of $g$ loops, such that they dissect $S$ into a sphere with $2 g$ deleted discs. It is clear that the normalizer of $\mathcal{N}$ is also $\mathcal{M}$. In particular, $\mathcal{M}(\mathcal{N})$ defines a Schottky group $G$, up to isomorphisms, and in particular, a Schottky uniformization $(\Omega(G), G, \pi: \Omega(G) \rightarrow S)$, up to equivalence, of the surface $S$. Since the set of loops obtained, as a consequence of the above results, is invariant under the action of $H$, its normalizer $\mathcal{M}$ is also invariant under $H$; in particular, the Schottky uniformization defined by the family $\mathcal{M}$ loops is the desired one. This ends the proof of our theorem.

We must remark that this proof is not so informative as the proof given for the cyclic, $\mathbb{Z} / 2 \mathbb{Z} \oplus \mathbb{Z} / 2 \mathbb{Z}$ and dihedral cases done in $[\mathbf{8}, \mathbf{9}, \mathbf{1 0}]$.

\section{REFERENCES}

1. L. V. Ahlfors and L. Sario, Riemann surfaces (Princeton Univ. Press, 1960).

2. L. Bers, Automorphic forms for Schottky groups, Adv. in Math. 16 (1975), 332-361.

3. V. Chuckrow, On Schottky groups with applications to Kleinian groups. Ann. of Math. 88 (1968), 47-61.

4. H. Farkas and I. Kra, Riemann surfaces (Springer-Verlag, 1980).

5. R. Hidalgo, On $\gamma$-hyperelliptic Riemann surfaces, Preprintreihe Sonderforschungsbereich 343 "Discrete Structuren in der Mathematik" 91-045.

6. R. Hidalgo, On $\gamma$-hyperelliptic Schottky groups, Notas Soc. Mat. de Chile 8 (1989), 27-36.

7. R. Hidalgo, On Schottky groups with automorphisms (Thesis Ph.D. in Mathematics, S.U.N.Y. at Stony Brook, 1991).

8. R. Hidalgo, On Schottky groups with automorphisms, Preprintreihe Sonderforschungsbereich 343 "Discrete Structuren in der Mathematik", 91-088.

9. R. Hidalgo, Schottky uniformizations of closed Riemann surfaces with the Klein groups as conformal group of symmetries, Preprintreihe Sonderforschungsbereich 343 "Discrete Structuren in der Mathematik", 91-087. 
10. R. Hidalgo, Closed Riemann surfaces with Dihedral groups of Conformal Automorphisms, Preprintreihe Sonderforschungsbereich 343 "Discrete Structuren in der Mathematik", 91-086.

11. L. Keen, On hyperellptic Schottky groups, Ann. Acad. Sci. Fenn., series A.I. Mathematica 5 (1980), 165-174.

12. F. Klein, Neue Beiträge zur Riemann'schen Funktionentheorie, Math. Ann. 21 (1883), 141-218.

13. P. Koebe, Über die Uniformisierung der Algebraischen Kurven II, Math. Ann. 69 (1910), $1-81$.

14. B. Maskit, Kleinian groups (Grundlehren der Mathematischen Wissenschaften, vol. 287, Springer-Verlag, 1988).

15. B. Maskit, A characterization of Schottky groups, J. d'Analyse Math. 19 (1967), 227-230.

16. B. Maskit, Canonical domains on Riemann surfaces, Proc. of the Amer. Math. Soc. 106 (1989), 713-721.

17. B. Maskit, On the classification of Kleinian groups I and II, Acta Math. 135 (1975), 249-270 and 138 (1977), 17-42.

Departamento de Mathemáticas

UTFSM

VALPARAISO

CHILE 